\title{
Phase acquisition techniques for RFID multistatic setups
}

\author{
Guillermo Alvarez-Narciandi ${ }^{\mathrm{a}, *}$, Jaime Laviada ${ }^{\mathrm{a}}$, Marcos R. Pino ${ }^{\mathrm{a}}$, Fernando Las-Heras ${ }^{\mathrm{a}}$ \\ ${ }^{a}$ Área de Teoría de la Señal y Comunicaciones, Departamento de Ingeniería Eléctrica, Universidad de Oviedo, Spain
}

\begin{abstract}
The aim of this paper is to present two different schemes to retrieve the phase of the field scattered by a UHF RFID tag without requiring a physical connection between the transmitter and the receiver systems. Thus, the phase angle can be comfortably measured in a multistatic, easy to deploy RFID sensor network for different purposes such as location and imaging. The accuracy of both schemes is validated against the one achieved by resorting to a physical connection between the transmitter and the receiver, demonstrating an excellent accuracy. Finally, a localization scheme based on this acquired information is presented to illustrate the use of the proposed phase retrieval approaches.
\end{abstract}

Keywords: RFID; multistatic; phase retrieval; localization system.

\section{Introduction}

The identification of objects and people (for instance hospital patients or factory workers) and the capacity to obtain complementary information is essential in many applications such as security or supply chain management. In particular, the availability of the accurate location of an object, its attitude, and the

5 ability to track its position provide a great added value. In order to achieve that, it is necessary to deploy sensor networks in the area of interest. There are different sensing devices based on various principles such as visible light, infrared, ultrasounds or radiofrequency, which is able to work in non-line of sight situations. However, it is desirable to use cost effective hardware to enable the deployment of large sensor networks without very large investments.

A technology that suffices the previous requirements is Radio Frequency IDentification (RFID), which is widely spread for the identification of goods. Among the different RFID standards, the EPCglobal Gen2 [1] (defined for the UHF band) is very mature and a lot of research based on it has been carried out. The basic architecture of these systems consists of an RFID reader, which interrogates or queries the RFID tags, and RFID tags, which answer to the reader. There are several types of RFID tags (active, passive or semi-passive [2]), but the passive tags are of special interest since they do not have batteries and their cost is extremely

\footnotetext{
*Corresponding author

Email address: ganarciandi@tsc.uniovi.es (Guillermo Alvarez-Narciandi)
} 
low (euro cents). Although the read range of passive tags is reduced to less than $10 \mathrm{~m}$, they are powered up and backscatter the information to the receiver with the energy of the signal of the reader. In addition, they hardly require maintenance.

In this context, several passive-RFID location systems have been proposed. Most of them are based on received signal strength (RSS) measurements, as this is the parameter provided by most commercial readers for monostatic as well as multistatic configurations. Among these systems, there are some that consist of deploying RFID tags in the test scenario and mounting one or more readers in the mobile assets. The accuracy of such systems depends mainly on the density of deployed tags [3]. In other RSS-based systems, the objects to be tracked are equipped with RFID tags and several readers are deployed [4, 5, 6]. However, RSS measurements, especially in indoor environments, are subject to fluctuations due to multipath propagation which lead to an accuracy reduction. There are other systems based on phase measurements of the signal backscattered by RFID tags. That information can be used to triangulate the position of the tags $[7,8,9]$ or to create synthetic apertures such us in [10], where a reader (whose trajectory must be known) is moved while it queries the RFID tags to be located, or inverse synthetic apertures as presented in $[11,12]$ (where an RFID reader is static whilst a tag, whose trajectory must be known, is moving). To the best authors' knowledge, these parameters are only provided by some of the most modern readers and only available for monostatic setups. Nevertheless, it has been shown that this information can be exploited to improve already available setups. In addition, the performance of these systems can be enhanced by using several carrier frequencies.

Only little research has been done using bistatic approaches based on either RSS [13] or phase measurements $[14,15]$. This approach can be advantageous in some situations in terms of flexibility of the setup, the obtained phase measurements (combining information from the direct path between antennas and the path antenna-tag-antenna) and the potential application of other techniques such aus tomography to enable advanced device-free user localization, where the users do not cooperate with the location sensing network, i.e., they do not carry any device of the system such as an RFID tag.

A different approach is proposed in [16], where apart from RFID readers and RFID tags, there is a third kind of devices called RFID listeners. These devices do not play an active role in the communication, they decode the messages between the reader and the tag. However, phase retrieval information has not been exploited by these devices. In fact, they are mostly based on ASK demodulation schemes. Finally, there are some hybrid systems that combine RFID with other technologies such as UltraWide-Band (UWB) [17].

The main contribution of this work is the demonstration of the capability to recover the phase of the signal backscattered by an RFID tag using only the over the air received signal for multistatic setups. Also, the unambiguous phase area achieved by these setups, when compared to the monostatic ones, is exploited to tailor a positioning system with centimetric accuracy to show a potential application of the presented phase recovery schemes. 
The paper is structured as follows. The different phase retrieval schemes are presented in Section 2. In Section 3 the measurement setups are described and the obtained results are discussed. Finally, in Section 4 the localization system developed to illustrate a potential application of multistatic phase measurements is presented.

\section{Phase Acquisition Schemes}

\subsection{Tag phase model for a given bistatic combination}

Modern commercial readers are able to provide the phase angle of the signal backscattered by an RFID tag, usually referred to as tag phase, which is acquired by comparing the phase of the field backscattered by the tag with the phase of the local oscillator, which generates the wave to be modulated by the tag. However, this situation cannot be assumed if there is not a physical connection between the transmitter and the receiver as in the case of multistatic setups. Thus, the tag phase for these setups must be firstly defined.

In this paper, for each transmitter and receiver combination, the phase angle, $\Phi(\vec{r})$, is defined as the phase difference between the signal transmitted directly from the transmitter to the receiver and the signal backscattered by the RFID tag. In addition, we will only focus on the case of RFID tags using an ASK scheme, which is the most popular among the multiple ones considered in the standard for UHF RFID [1].

The paths of the signal transmitted by the reader, $s_{r}$, and the signal backscattered by the tag, $s_{\text {tag }}$, are depicted in Fig. 1 for a given transmitter and receiver pair. The length of the direct path between the transmitter and the receiver is denoted by $R_{D} . R_{T}$ stands for the length of the path from the transmitter to the RFID tag and $R_{R}$ is the distance from the RFID tag to the receiver.

Figure 1: Relevant parameters for the definition of the phase angle for a given transmitter and receiver combination.

In this setup, $\Phi(\vec{r})$ is proportional to the difference between the direct path transmitter-receiver $\left(R_{D}\right)$ 
and path of the carrier sent by the reader to the tag $\left(R_{T}\right)$ and the path of its backscattered signal to the receiver $\left(R_{R}\right)$ and is given by:

$$
\Phi(\vec{r})=\left(\frac{2 \pi}{\lambda} R_{D}+\varphi_{T x-R x}\right)-\left(\frac{2 \pi}{\lambda}\left[R_{T}(\vec{r})+R_{R}(\vec{r})\right]+\varphi_{T x-t a g}(\vec{r})+\varphi_{t a g-R x}(\vec{r})\right)+\varphi_{0},
$$

where $\vec{r}$ denotes the position of the tag and $\lambda$ is the wavelength. The terms inside the first parenthesis account for the propagation delay of the carrier sent by the reader to the receiver and for the phase term introduced due to the radiaton pattern of both the transmitting and receiving antennas, $\varphi_{T x-R x}$. The terms in the second parenthesis account for the propagation delay of the signal sent by the reader to the tag (by means of $R_{T}(\vec{r})$ ), for the propagation delay of the signal backscattered by the tag to the receiving antenna (by means of $R_{R}(\vec{r})$ ) and for the phase terms introduced by the transmitting antenna, the receiving antenna and the tag antenna: $\varphi_{T x-\operatorname{tag}}(\vec{r})+\varphi_{\text {tag-Rx }}(\vec{r})$ [7]. Finally, $\varphi_{0}$ is the phase offset introduced by the RFID tag.

Since the RFID tag is expected to be in the coverage area provided by the main lobe of the antennas, where the phase is typically constant, and assuming that the phase difference due to the tag orientation is negligible, the previous model can be simplified as:

$$
\Phi(\vec{r})=-\frac{2 \pi}{\lambda}\left(R_{T}(\vec{r})+R_{R}(\vec{r})-R_{D}\right)+\varphi_{0} .
$$

It is relevant to notice that the iso-phase surfaces given by (2) are ellipsoids whose foci are the transmitter and the receiver.

Next, the two proposed approaches to retrieved the tag phase based on the over the air received RFID signal are presented. In addition, a reference setup, providing equivalent information but based on a wired connection, is also presented.

\subsection{Delayed version of the received signal}

According to the Gen 2 standard, after the reader sends a query frame, it keeps sending a monochromatic wave at the working frequency. After a given period of time, the RFID tag starts its response by changing the input impedance of its antenna so that the amplitude of the monochromatic wave is modulated. Thus, as shown in the phasor diagram of Fig. 2, the received signal is the vector sum of the signal transmitted by the reader and the signal backscattered by the RFID tag. As a consequence, when the RFID tag uses ASK modulation and encodes a low state, the received signal, $s_{r x}$, overlaps with the signal transmitted by the reader. Hence, it is possible to obtain the tag phase by comparing the phase of $s_{r x}$ during the two possible states of the reply of the tag. 


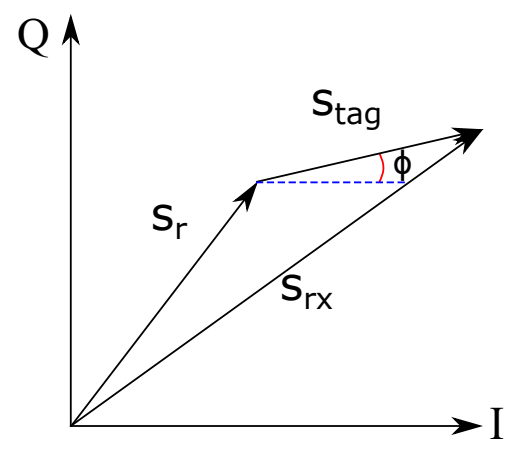

Figure 2: Phasor diagram of the received signal, $s_{r x}$, which is a vector sum of the signal transmitted by the reader, $s_{r}$, and the signal backscattered by the RFID tag, stag.

In order to further clarify this approach, the signals involved in the demodulation process are plotted in Fig. 3. First, in the upper part, a fragment of the baseband signal of the signal backscattered by the tag is represented, where Tari is the reference time interval for a ' 0 ' symbol. The duration of the Tari is specified by the reader in its query according to the standard [1]. Below the baseband signal, the carrier emitted by the reader, of amplitude $A$, is represented. In the middle, the received signal, $s_{r}+s_{\text {tag }}$, where $B$ (whose value has been exaggerated for the ease of interpretation) is the amplitude of the signal backscattered by the tag, is plotted. Finally, at the bottom, a Tari/2 delayed version of the received signal, which is used as local oscillator, is depicted. As can be seen, during the high states of the baseband signal (enclosed with dotted red line), the delayed version of the received signal is coincident with $s_{r}$ and therefore, it can be used to demodulate $s_{r x}$.

During the first part of the preamble of the signals backscattered by an RFID tag there is a chain, which contains the symbol ' 0 ' repeated a different number of times depending on the encoding selected by the reader [1]. The symbol ' 0 ' is encoded with a high state during half of the Tari and the other half is encoded with a low state. Thus, the standard guarantees that this method can be successfully applied for the different tag messages.

This approach enables the implementation of a wireless multistatic RFID system, as can be seen in the block diagram represented in Fig. 4, but a delay of several microseconds at a frequency around $866 \mathrm{MHz}$ must be introduced. This delay can be achieved by means of digital signal processing, but it would require a high sampling rate as well as relatively large buffers.

Alternatively, large delays can be obtained by using a Surface Acoustic Wave (SAW) delay line. However, the insertion losses of these devices at the UHF RFID frequency band are considerably high $(\sim 26 \mathrm{~dB})$ [18]. In addition, the delay line should be changed if the modulation parameters of the RFID reader are modified. As a result, the system would be less flexible. 


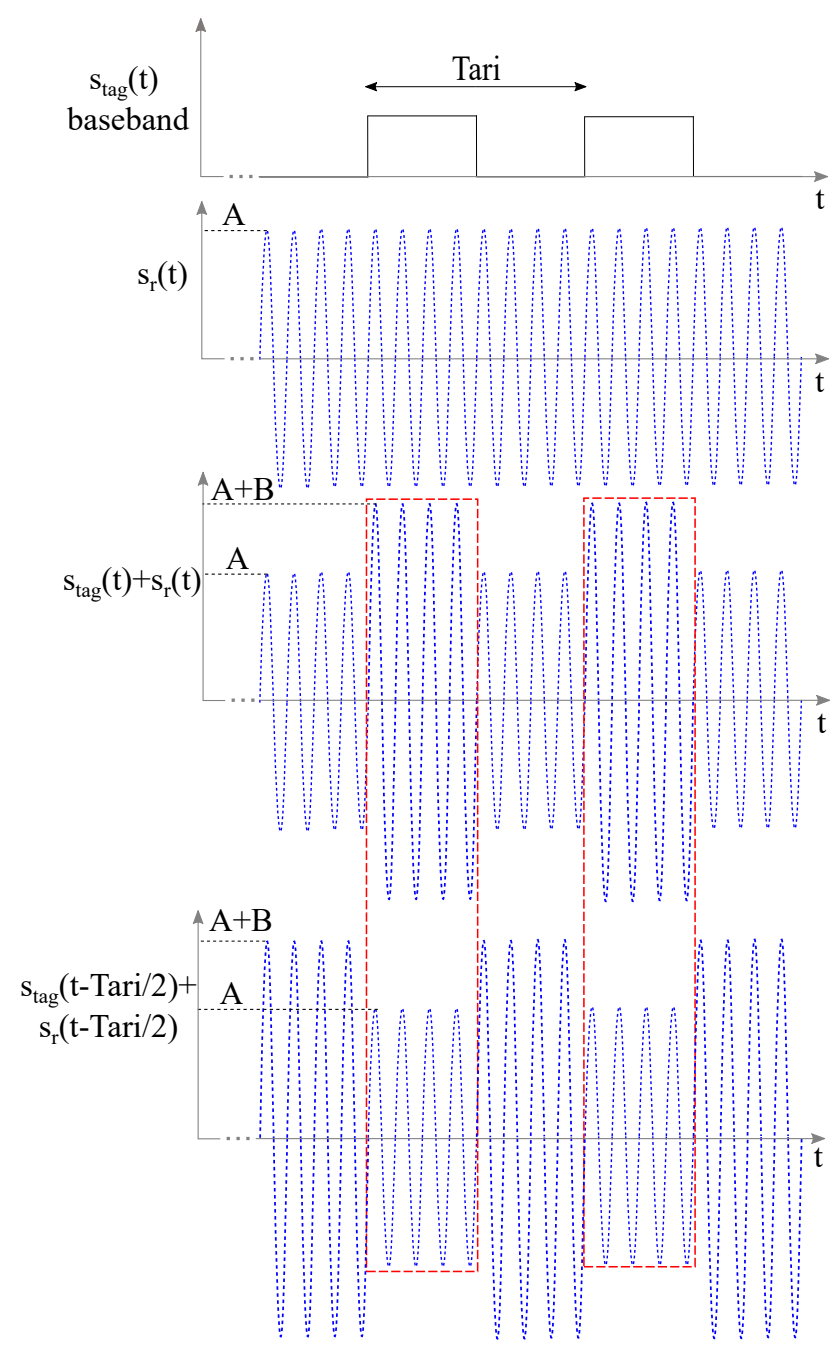

Figure 3: Scheme of the demodulation of the received signal based on delaying it Tari/2 to use it as local oscillator.

\subsection{Using a Phase Lock Loop}

The last approach consists of using a phase lock loop (PLL) at the receiver. The PLL should lock to the frequency of the carrier emitted by the reader but it should not track the small and short frequency variations of the backscattered signal. Hence, it would be possible to use the output signal of the PLL as local oscillator to demodulate the received signal. The block diagram of this approach is illustrated in Fig. 5.

This scheme to demodulate the received signal and retrieve its phase allows the avoidance of wires between transmitter and receiver. Additionally, it simplifies the hardware required with respect to the second approach or, as well as the second approach, it can be implemented via software. 


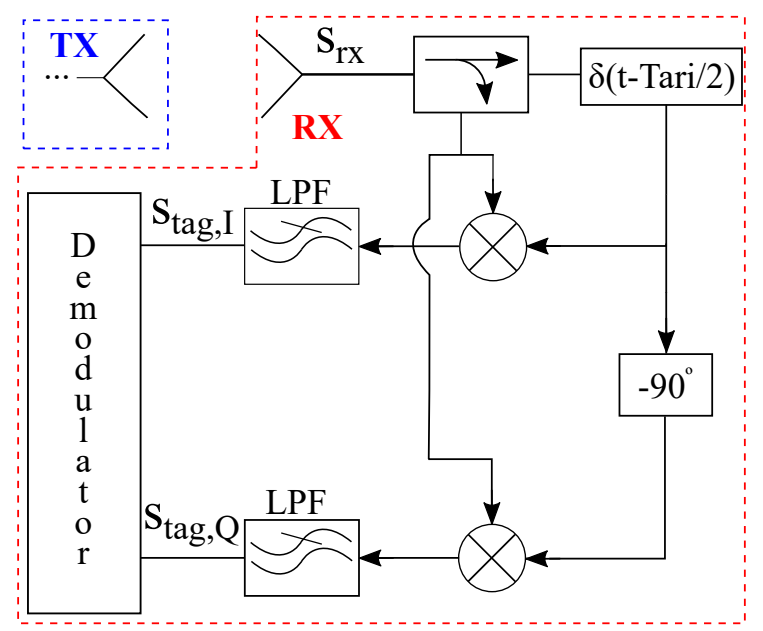

Figure 4: Block diagram of the phase retrieval scheme based on a delayed version of the received signal.

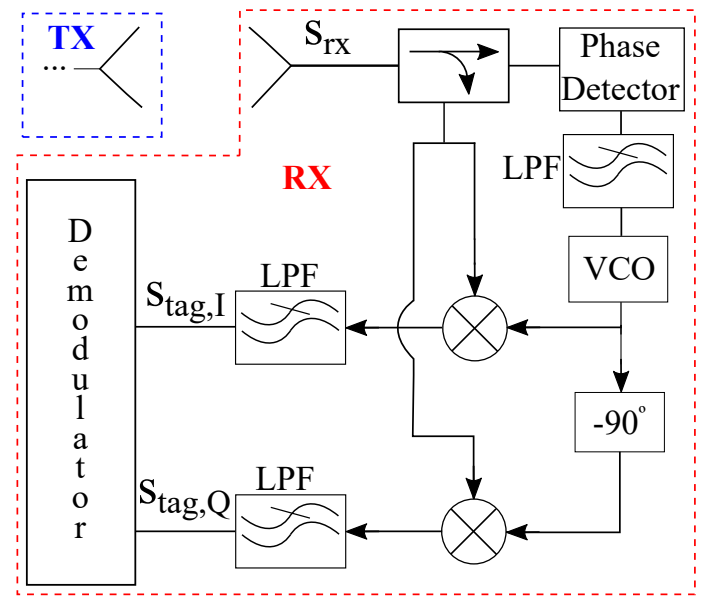

Figure 5: Block diagram of the phase retrieval scheme based on using a PLL.

\subsection{Reference phase acquisition setup}

This reference scheme is based on the direct connection between the transmitter and receiver as shown in Fig. 6.

Thus, the carrier signal transmitted by the RFID reader is connected to a IQ demodulator, where it is used as the local oscillator signal to obtain the IQ components of the backscattered signal.

It is important to notice that this setup introduces some new delays such as the one introduced from the directional coupler to the IQ mixer (that depends on the electrical length of the used transmission line). Nevertheless, these are fixed delays and, therefore, the dependence of the tag phase with the tag position is still ruled by (2).

Despite the relative simplicity of this scheme, it also imposes several constraints in terms of hardware. 


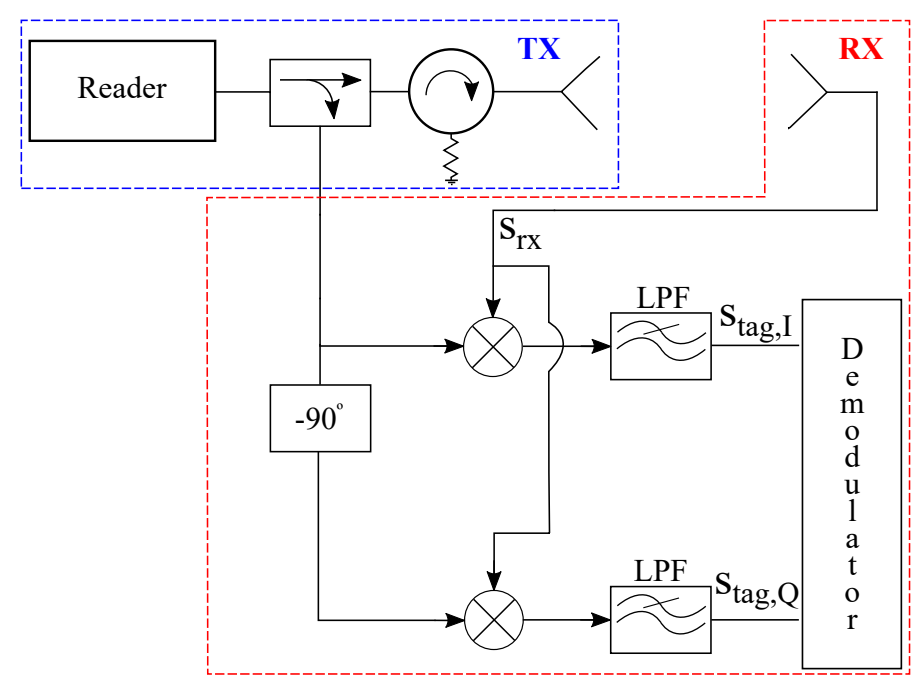

Figure 6: Block diagram of the phase retrieval scheme based on connecting transmitter and receiver through a wire.

this setup.

\section{Phase acquisition for a single $T x / R x$ link}

In this section, the results of the phase measurements obtained with the aforementioned approaches are validated for a single transmitter and receiver combination as depicted in Fig. 1. The Speedway ${ }^{\circledR}$ Revolution Reader [19] of $\operatorname{Impinj}{ }^{\circledR}$ was used to generate the interrogating signals for all the measurements. The PLL was implemented using a damping factor $\xi=0.6$, a pull-in range of $2 \mathrm{MHz}$, a phase detector of gain $k_{p}=1$, a second order loop filter and a local oscillator with $\omega_{0}=866 \mathrm{MHz}$ and whose gain was set to $k_{0}=0.003$. In order to test the proposed techniques, they were implemented via software. Specifically, both the signal of the reader, $s_{r}$, and the received signal, $s_{r x}$, were recorded by means of the digital oscilloscope Tektronix CSA7404 using a sampling rate of 10 of gigasamples per second. After that, the signals were interpolated by a factor of 4 and the following steps were performed to estimate the tag phase:

1. First, the IQ components of the received signal are computed using the oscillator signal obtained with one of the schemes presented in Section 2. After low-pass filtering, the IQ components, depicted in Fig. 7 , are given by:

$$
\begin{aligned}
s_{I}(t) & =\left[\left(s_{\text {tag }}(t)+s_{r}(t)\right) \cos \left(\omega_{c} t+\Phi_{O s c}\right)\right] * h_{L P F}(t) \approx \\
& \approx \frac{A}{2} \cos \left(\Phi_{r}-\Phi_{O s c}\right)+\frac{B}{2} x(t) \cos \left(\Phi_{\text {tag }}-\Phi_{O s c}\right)+n_{I}(t)
\end{aligned}
$$

and

$$
\begin{aligned}
s_{Q}(t) & =-\left[\left(s_{\text {tag }}(t)+s_{r}(t)\right) \sin \left(\omega_{c} t+\Phi_{O s c}\right)\right] * h_{L P F}(t) \approx \\
& \approx \frac{A}{2} \sin \left(\Phi_{r}-\Phi_{O s c}\right)+\frac{B}{2} x(t) \sin \left(\Phi_{\text {tag }}-\Phi_{O s c}\right)-n_{Q}(t)
\end{aligned}
$$




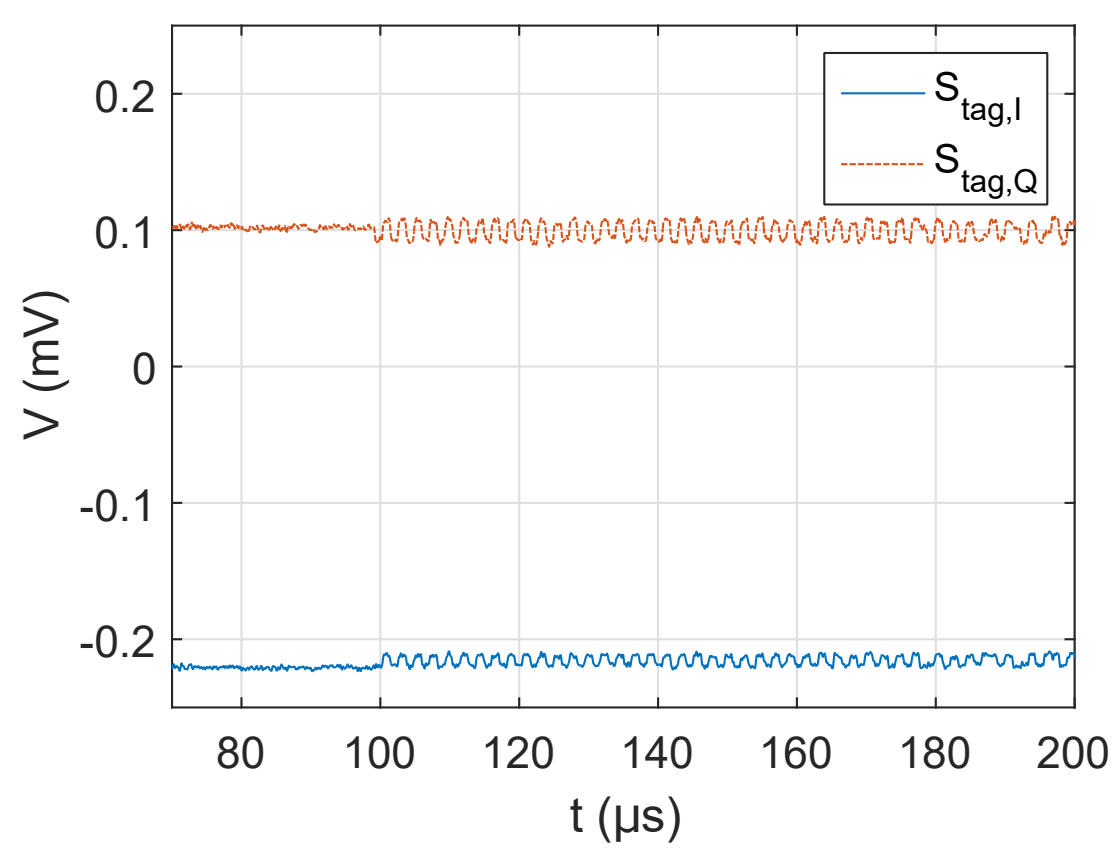

Figure 7: IQ components of the signal backscattered by the tag obtained after performing step 1 .

wherein $A$ is the amplitude of the monochromatic wave emitted by the transmitter and $B$ is the amplitude of the signal backscattered by the tag (in general, $A \gg B$ ); $\omega_{c}$ is the angular carrier frequency; $x(t)$ is the baseband signal of the signal backscattered by the tag; $\Phi_{r}$ is the phase of the signal sent by the reader $s_{r}$; $\Phi_{t a g}$ is the phase of the signal backscattered by the tag, $s_{t a g}$; $\Phi_{O s c}$ is the phase of the regenerated local oscillator signal at the receiver; and $n_{I}(t)$ and $n_{Q}(t)$ are the noise contributions of the IQ components of the signal. The low pass filter, whose impulse response is $h_{L P F}(t)$, is a Butterworth filter of order 5 and cut-off frequency of $20 \mathrm{MHz}$.

2. The offset levels of the I and Q components, which are given by

$$
\text { Offset }_{I}=\frac{A}{2} \cos \left(\Phi_{r}-\Phi_{\text {Osc }}\right)
$$

and

$$
\operatorname{Offset}_{Q}=\frac{A}{2} \sin \left(\Phi_{r}-\Phi_{O s c}\right)
$$

respectively, are computed and subtracted from the I and Q components. As can be observed, both are proportionaldepend on a trigonometric function (cosine and sine for I and Q componentes respectively) whose argument is to the difference between the phase of the monochromatic signal emitted by the reader, $\Phi_{r}$, and the phase of the oscillator signal which was used to demodulate the received signal, $\Phi_{O s c}$. Therefore, the phase difference $\Phi_{r}-\Phi_{O s c}$ can be obtained by means of a two-argument arctangent operation. 


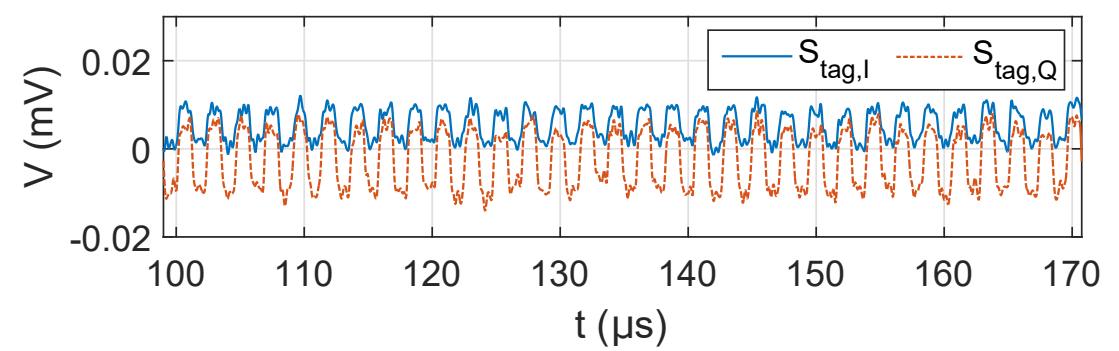

Figure 8: First part of the preamble of the IQ components of the signal backscattered by the tag.

3. The beginning of the preamble of the backscattered signal (Fig. 8) is computed correlating the IQ components obtained in step 2, i.e. without their offset levels, with the preamble defined by the reader in its query.

4. Once the beginning of the preamble is estimated, the position of the high states of the preamble of the signal backscattered by the tag is known. Thus, the phase term $\Phi_{t a g}-\Phi_{O s c}$ can be obtained by means of a two-argument arctangent operation applied during the high states of the preamble of the IQ components. This provides an instantaneous tag phase value which is averaged along the acquisition time. Finally, the desired phase angle, $\Phi(\vec{r})=\Phi_{t a g}-\Phi_{r}$, can be computed subtracting the phase terms estimated during the steps 2 and 4 .

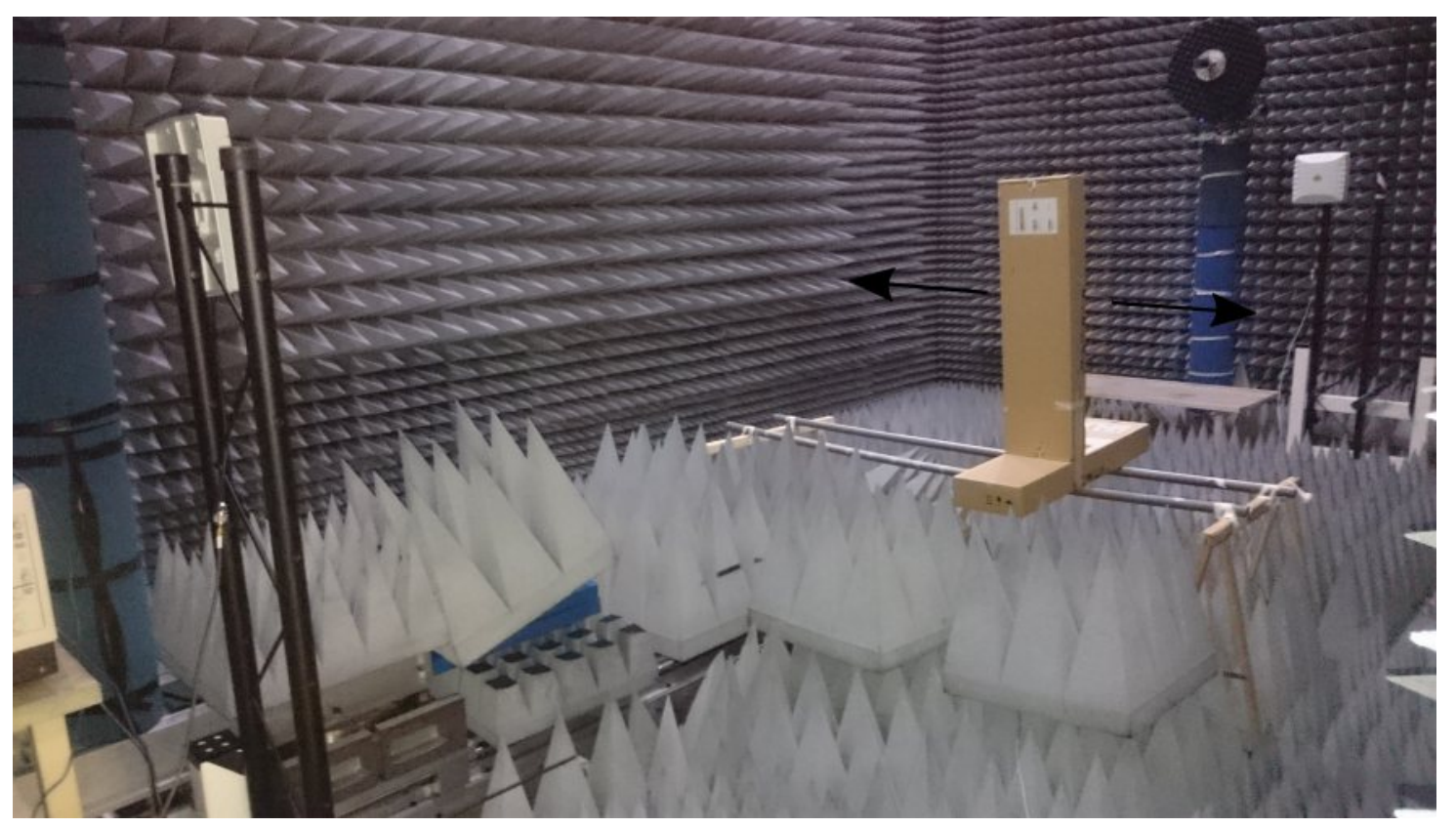

Figure 9: Measurement setup in the anechoic chamber. 
Signal Theory and Communications of the University of Oviedo. Second, the proposed approaches were tested in an indoor scenario. The setup in the anechoic chamber is shown in Fig. 9, where two antennas were placed opposite to each other at a distance of $4.5 \mathrm{~m}$. In the middle of the imaginary line between both antennas, a structure of two PVC tubes was used as rails to move a cardboard box with an attached RFID tag. During the measurements the RFID tag was moved from $-60 \mathrm{~cm}$ to $60 \mathrm{~cm}$ with $4 \mathrm{~cm}$ steps. A line laser was used to align the initial position of the tag right across the line between the two antennas. In addition, the term $\varphi_{0}$ was previously measured and removed so that the theoretical phase values and the ones measured using the reference phase acquisition technique were both $\Phi=0$ at the center position $(d=0)$.

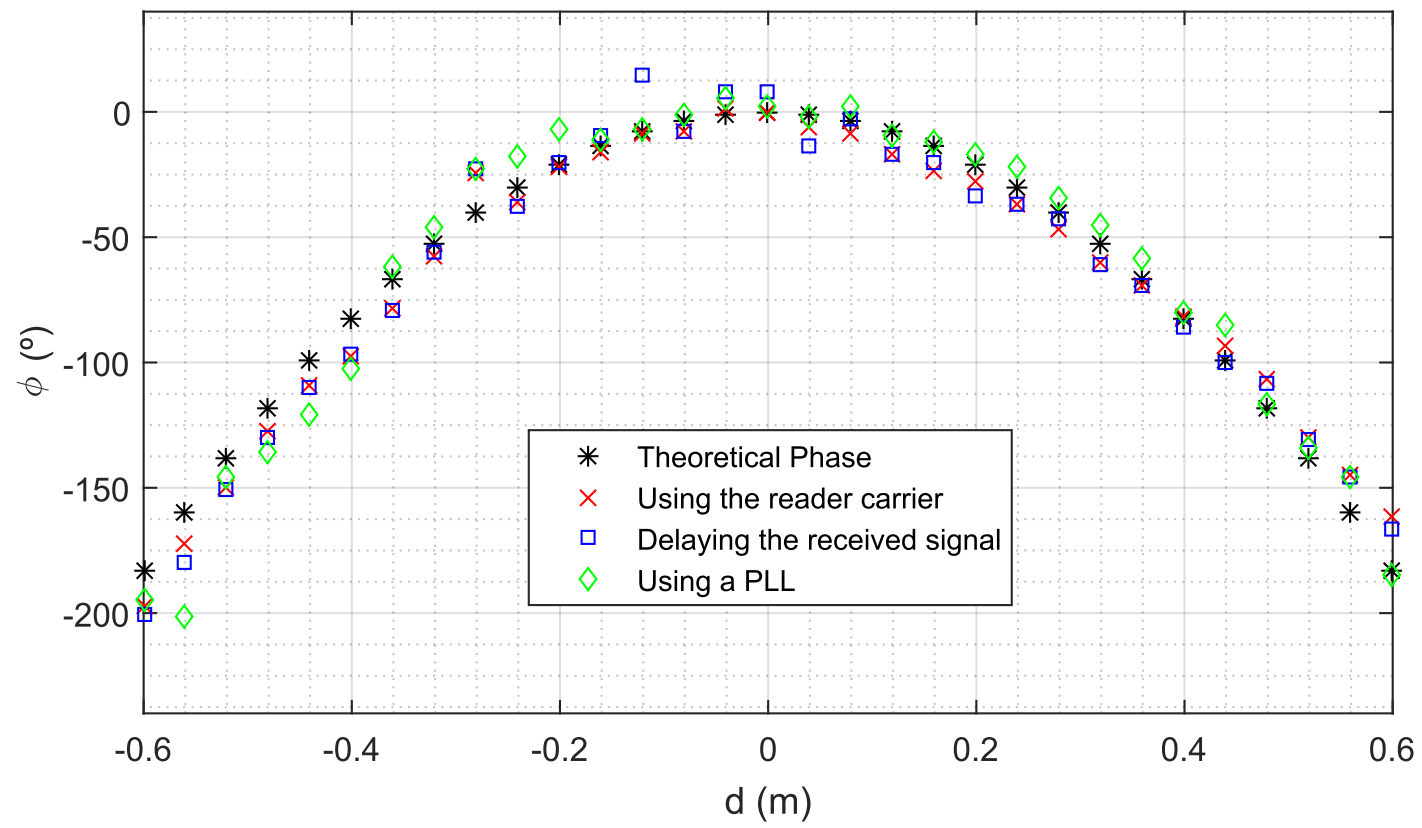

Figure 10: Retrieved phase of the signal backscattered by the tag measured in anechoic chamber using the presented approaches and the theoretical values.

The phase acquired employing the two approaches (using a PLL and delaying the received signal) is plotted in Fig. 10. The theoretical curve given by (2) and the empirical results given by the reference setup (i.e., using the reader carrier) are also depicted in that figure. As can be seen, there is a good agreement between the empirical and theoretical results. The root mean square errors, computed comparing the obtained phase values using each phase retrieval scheme and the theoretical ones, are 7.94, 9.37 and 8.80 degrees for the case of using the reader carrier, delaying the received signal and using a PLL, respectively. If instead of comparing the phase values obtained using the proposed wireless techniques with the theoretical ones, we compare them with the phase values obtained with the reference phase acquisition technique (to account for small positioning errors) the root mean square errors are 5.77 degrees when delaying the received 


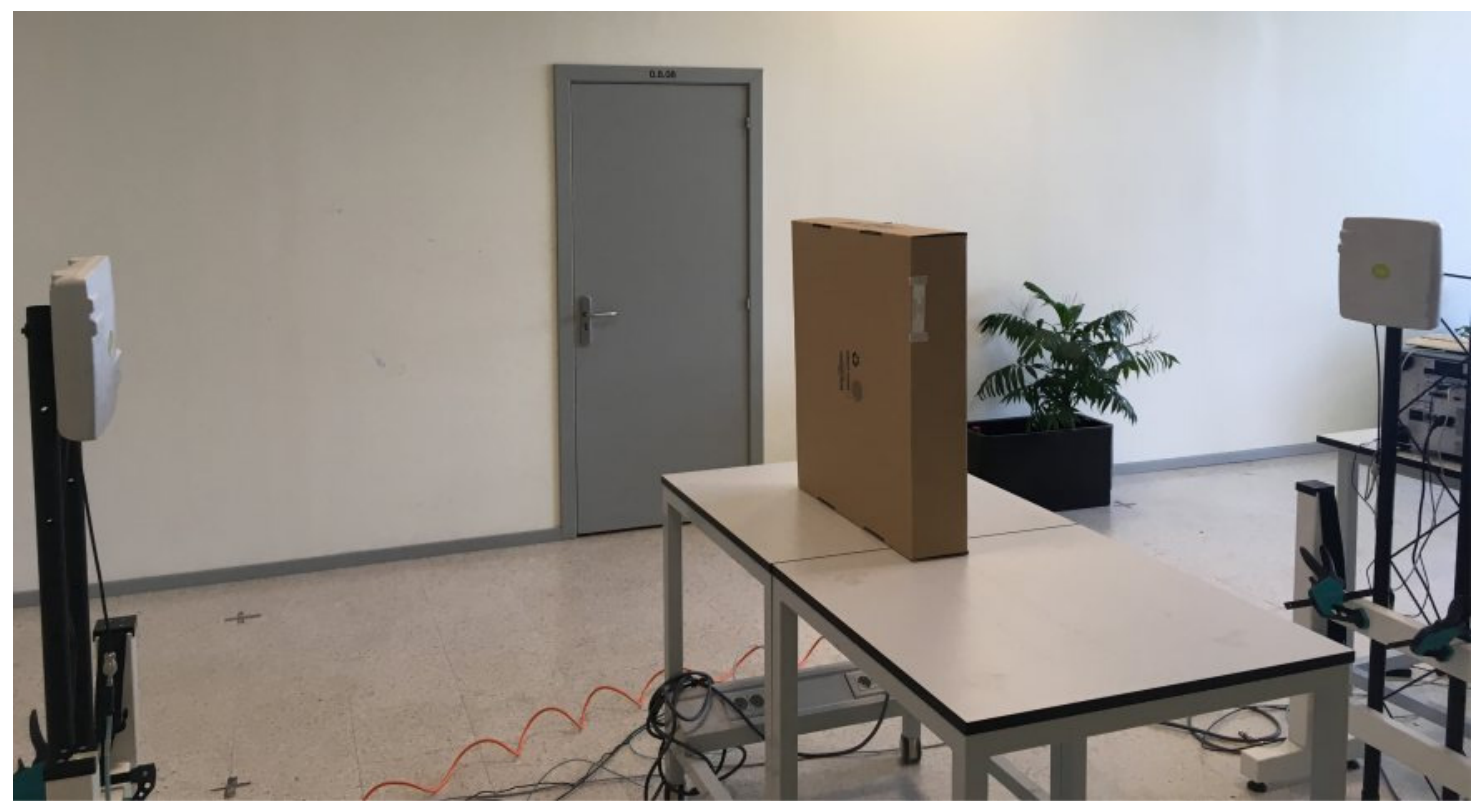

Figure 11: Measurement setup in an indoor scenario.

The setup in the indoor scenario is shown in Fig. 11, where the two antennas were placed opposite to each other at a distance of $3 \mathrm{~m}$. In the middle of the imaginary line between both antennas, a cardboard box with an RFID tag attached to it was placed over a table. During the measurements, the RFID tag was moved from $-60 \mathrm{~cm}$ to $60 \mathrm{~cm}$ with $4 \mathrm{~cm}$ steps (as in the anechoic chamber measurements). A line laser was true ones, and comparing them with the other two wireless phase retrieval schemes. Using this metric, the root mean square errors are 8.26 and 13.11 degrees for the case of delaying the received signal and using a PLL, respectively. Thus, results show that, although the performance of the phase retrieval schemes is 


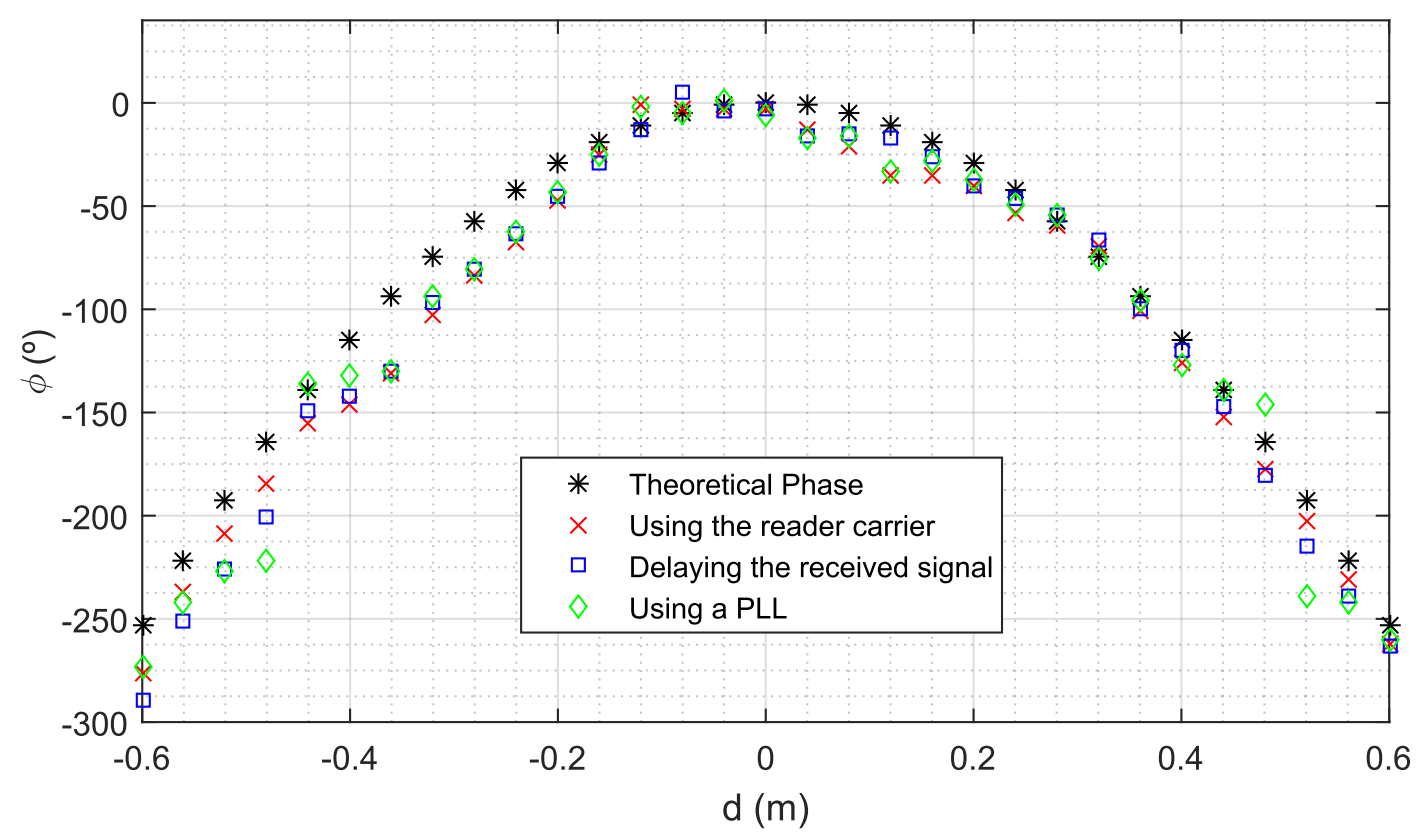

Figure 12: Retrieved phase of the signal backscattered by the RFID tag measured in an indoor scenario using the presented approaches and the theoretical values.

slightly degraded in an indoor scenario, the proposed approaches are still accurate.

\section{Application to a positioning system}

In order to illustrate the capabilities of multistatic phase measurements of the signal backscattered by an RFID tag, an indoor location system was developed. One of the limitations of traditional location systems that use (multi)monostatic phase measurements is the inherent $2 \pi$ ambiguity of phase. In contrast, although multistatic systems are also subject to phase ambiguity, the phase variation is slower with the distance, (1), especially in the region between the antennas and, therefore, the unambiguous region is extended.

This is shown in Fig. 13, where the phase which would be measured with a monostatic setup (Fig. 13a) and with a bistatic setup (Fig. 13b) for several positions of a tag is represented. The phase value for each position was computed assuming free space propagation from the transmitting antenna to the tag and back to the transmitting antenna again in the monostatic case and to the receiving antenna in the bistatic case. In the monostatic case, where the antenna is located at $(-2,0) \mathrm{m}$, there is phase ambiguity for those positions located at a distance $l>\lambda / 2$ from the antenna taking into account both the direct and the reverse path of the signal which, at the UHF RFID European band, is approximately $17.3 \mathrm{~cm}$. In contrast, in the bistatic case, the unambiguous zone is enclosed by an ellipse (an ellipsoid in a 3D space) whose foci are the 


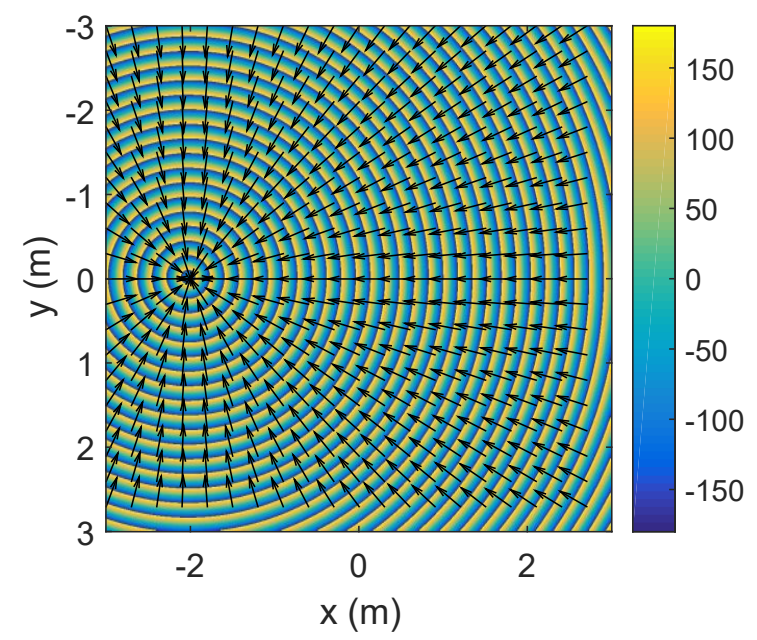

(a)

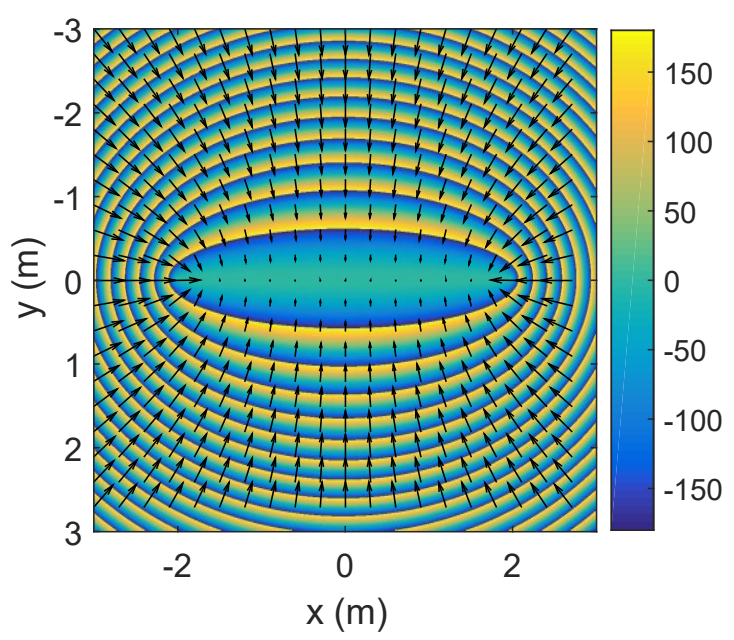

(b)

Figure 13: Tag phase distribution (in degrees) at $866 \mathrm{MHz}$ for : a) monostatic setup with an antenna at $(-2,0)$ m; b) bistatic setup with antennas at $(-2,0) \mathrm{m}$ and $(2,0) \mathrm{m}$. The black arrows depict its gradient.

two antennas (separated $R_{D}$ ), whose semi-major axis is given by

$$
a=\frac{R_{D}}{2}+\frac{\lambda}{2}
$$

and whose semi-minor axis is given by

$$
b=\sqrt{R_{D} \frac{\lambda}{2}}+\frac{\lambda^{2}}{4}
$$

Therefore, the unambiguous phase area in a bistatic setup, assuming that the antennas do not radiate backwards, is

$$
A=2 a b\left[\arcsin \left(\frac{R_{D}}{2 a}\right)-\frac{\sin \left(2 \arcsin \left(\frac{R_{D}}{2 a}\right)\right)}{2}\right],
$$

which, placing the antennas at $(-2,0) \mathrm{m}$ and $(2,0) \mathrm{m}$ and transmitting at $f=866 \mathrm{MHz}$, is approximately 120 times bigger than in a monostatic setup. The main drawback of the bistatic setup is the sensitivity variation, but this can be overcome using more than two antennas to tailor a multistatic setup.

Based on this approach an indoor localization system which uses several antennas was designed. When each of the antennas of the setup queries an RFID tag, the phase of the signal backscattered by that tag is measured at the rest of the antennas (except the ones adjacent to the transmitter, so that the position of 


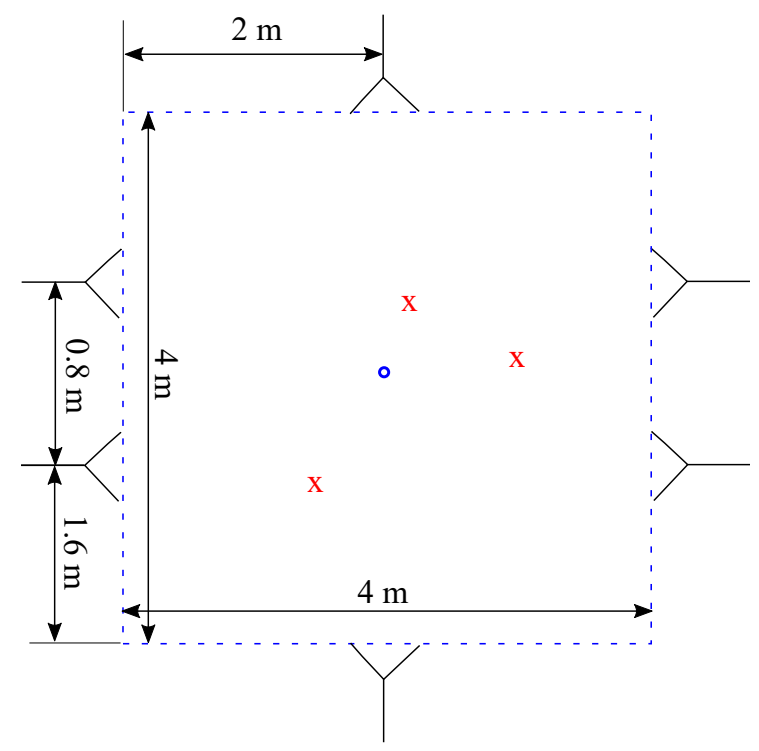

(a)

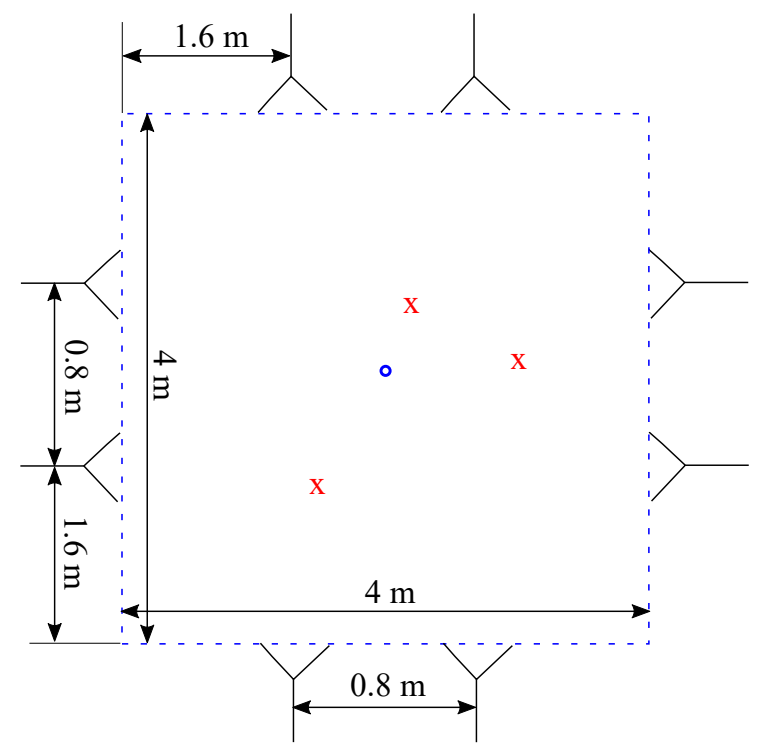

(b)

Figure 14: Schematic of the simulation setup using 6 (a) and 8 (b) antennas.

RFID tag is estimated as the one that makes the following cost function minimum

$$
J=\sum_{n=1}^{N \cdot(N-3) / 2}\left|\angle e^{j\left(\Phi_{\text {meas }, n}-\Phi_{\text {theor }, n}\right)}\right|^{2},
$$

where $N$ is the number of antennas and $\Phi_{\text {meas }, n}$ and $\Phi_{\text {theor }, n}$ are the measured and theoretical phases for the $n$-th transmitter-receiver antenna pair. To compute the theoretical phases, the area enclosed by the antennas is first discretized. Then, the phase values are computed assuming free space propagation as it was done to obtain Fig. 13b. The upper bound of summation $(N \cdot(N-3) / 2)$ is the number of diagonals of a polygon of $N$ sides, i.e., the number of bistatic combinations using $N$ antennas (without considering adjacent antennas). As can be seen, it is necessary to know the position of the antennas with accuracy to precompute the theoretical phase at each point of the grid. In addition, the phase introduced by the tag $\varphi_{0}$ must be firstly measured in a calibration stage so that it can be subtracted from the tag phase estimation.

In order to evaluate the performance of the proposed location system for different configurations, several simulations were carried out. In particular, the two setups shown in Fig. 14, with 6 and 8 antennas have been considered. As can be observed, the antennas enclose an area of $16 \mathrm{~m}^{2}$. The red crosses denote the positions of the simulated RFID tags whilst the blue circle indicates the center of the coordinate system.

The results after performing 20 simulations for each position of the RFID tag for the two different setups are summarized in Table 1, 2 and 3. The parameter $\sigma_{\text {noise }}$ denotes the standard deviation in degrees of the simulated phase measurements from the true phase value (althoug the used $\sigma_{\text {noise }}$ may seem high, they are 
realistic due to the features of RFID technology and its low cost nature, as shown in Section 3) and $\epsilon_{r m s}$ the position root mean square error in meters.

Table 1: Position root mean square error obtained for different values of $\sigma_{\text {noise }}$ simulating an RFID tag at $(0.2,0.5) \mathrm{m}$.

\begin{tabular}{|c|c|c|c|c|c|c|c|}
\hline \#Antennas & \multicolumn{4}{|c|}{6} & \multicolumn{3}{c|}{8} \\
\hline$\sigma_{\text {noise }}\left({ }^{\circ}\right)$ & 20 & 30 & 40 & 50 & 30 & 40 & 50 \\
\hline$\epsilon_{\text {rms }}(\mathrm{m})$ & 0.20 & 0.53 & 0.82 & 1.37 & 0.01 & 0.04 & 0.26 \\
\hline
\end{tabular}

Table 2: Position root mean square error obtained for different values of $\sigma_{\text {noise }}$ simulating an RFID tag at $(-0.4,-1) \mathrm{m}$.

\begin{tabular}{|c|c|c|c|c|c|c|c|}
\hline \#Antennas & \multicolumn{4}{|c|}{6} & \multicolumn{3}{c|}{8} \\
\hline$\sigma_{\text {noise }}\left({ }^{\circ}\right)$ & 20 & 30 & 40 & 50 & 30 & 40 & 50 \\
\hline$\epsilon_{\text {rms }}(\mathrm{m})$ & 0.01 & 0.33 & 1.11 & 1.74 & 0.05 & 0.13 & 0.37 \\
\hline
\end{tabular}

Table 3: Position root mean square error obtained for different values of $\sigma_{\text {noise }}$ simulating an RFID tag at $(1,-0.1) \mathrm{m}$.

\begin{tabular}{|c|c|c|c|c|c|c|c|}
\hline \#Antennas & \multicolumn{4}{|c|}{6} & \multicolumn{3}{c|}{8} \\
\hline$\sigma_{\text {noise }}\left({ }^{\circ}\right)$ & 20 & 30 & 40 & 50 & 30 & 40 & 50 \\
\hline$\epsilon_{\text {rms }}(\mathrm{m})$ & 0.20 & 0.62 & 1.11 & 1.60 & 0.01 & 0.07 & 0.33 \\
\hline
\end{tabular}

As expected, increasing the number of antennas entails a reduction of the positioning error. In fact, in the system was complemented by using the RSS of the signals backscattered by the tag in a monostatic setup, which does not imply the use of a more complex infrastructure. Hence, when an antenna queries the tag, the phase of the signal backscattered by it is measured at the other deployed antennas and its RSS is measured at the interrogator antenna. The information hybridization algorithm steps are the following: 


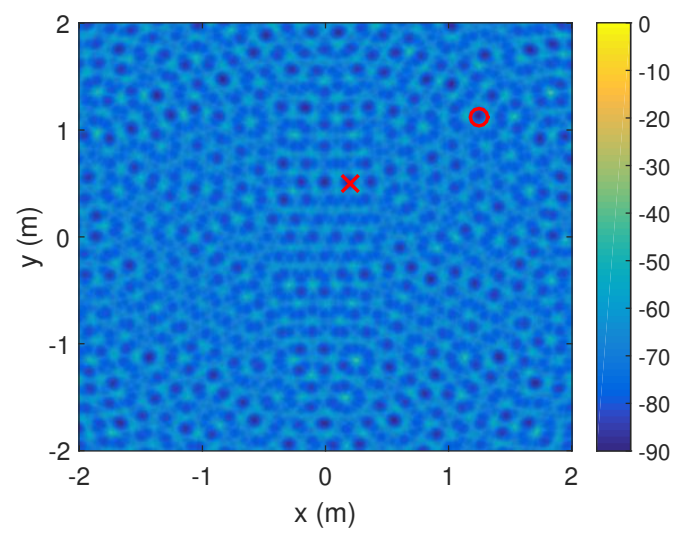

(a)

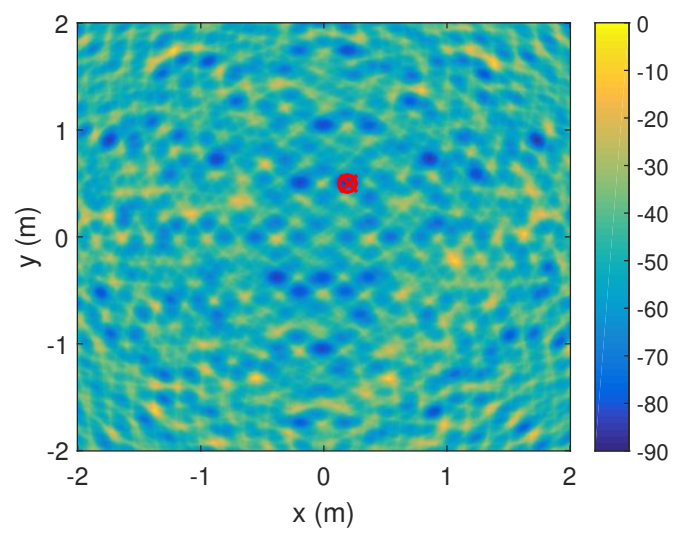

(b)

Figure 15: Cost function evaluated for the setup shown in Fig. 14a using a monostatic approach (a) and the proposed bistatic approach (b). The red circunmference depicts the simulated tag position and the red cross depicts the estimated tag position.

Table 4: Antenna coordinates of the localization system setup

\begin{tabular}{|c|c|c|c|c|c|c|}
\hline Antenna & 1 & 2 & 3 & 4 & 5 & 6 \\
\hline$x_{\text {coord }}(\mathrm{m})$ & 0.02 & 0.74 & 2.13 & 0.63 & 0.04 & -1.22 \\
\hline$y_{\text {coord }}(\mathrm{m})$ & 0 & 0 & 1.90 & 3.75 & 3.70 & 2.02 \\
\hline
\end{tabular}

1. A first estimate of the area where the RFID tag is located is performed using the RSS measurements. To do so, the algorithm proposed in Section 2.1 of [20], which is based on the attenuation differences between the antennas, is applied. Finally, the area of the RFID tag is limited within a circumference radius of $0.5 \mathrm{~m}$ and centered on the estimated position by the RSS algorithm.

2. Once the possible locations of the RFID tag are limited, the phase-based algorithm is applied. The reduction of the research domain eliminates local minima.

The results using multistatic phase measurements are shown in Fig. 17. In addition, the same positioning algorithm was used with monostatic phase measurements in order to compare the results of both schemes. As can be seen, despite a small offset in the order of $7 \mathrm{~cm}$, the accuracy of the position estimations using multistatic phase measurements is excellent, with a rms error of $8 \mathrm{~cm}$. On the other hand, the position estimations obtained with monostatic phase measurements are less accurate with a rms error of $35 \mathrm{~cm}$. Also, the estimations are less precise, i.e., the computed positions do not follow the pattern of the real positions of the tag (a straight line), the estimated positions are scattered (the variability of the position estimations is larger). It is worth mentioning that the stronger the multipath in an indoor scenario is, the more impact it would have in the accuracy of the localization system. However, based on the previous simulations and the measurement results of Section 3, it can be inferred that the accuracy of the system would still be high 


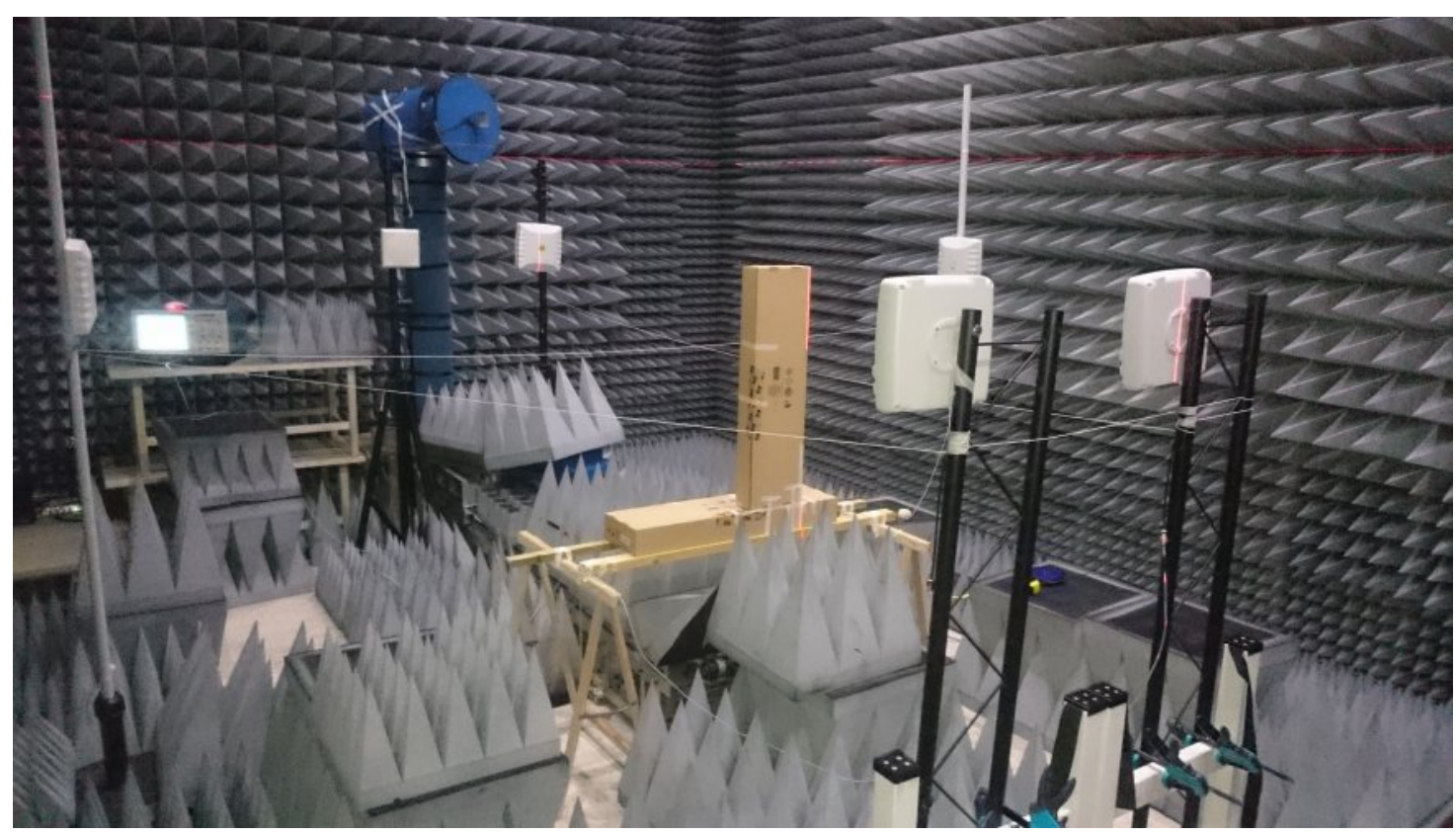

Figure 16: Setup of the proposed localization system.

in an indoor scenario.

\section{Conclusions}

It has been demonstrated in this paper that the phase angle of an RFID tag can be extracted from the over the air transmitted signal without the need of resorting to a physical connection between the transmitter and receiver. Thus, the phase information can be easily exploited to build advanced systems based on multistatic topologies.

For this purpose, two different phase acquisition schemes have been proposed with different advantages and drawbacks. The first one takes advantage of the properties of the RFID signal and it is based on demodulating using a Tari/2 delayed version of the received signal. Although this approach simplifies the setup, it implies the use of a delay line of a relatively high nominal delay for UHF frequencies (a SAW delay line) and whose insertion losses are very high. This also limits the encoding of the backscattered RFID signal (which modifies the value of Tari) to the one with the Tari value of the delay line. However, both disadvantages can be avoided if digital processing is used at the expense of using high sample rates and large data buffers. The second approach is based on the use of a PLL that can track the continuous wave emitted by the reader but it is slow enough so that it is not affected by the short periods when the tag reflects the impinging signal. This scheme is more flexible since it only uses the over the air transmitted signal and does not impose any constraints on the encoding used by the tag signal. Although both methods have been 


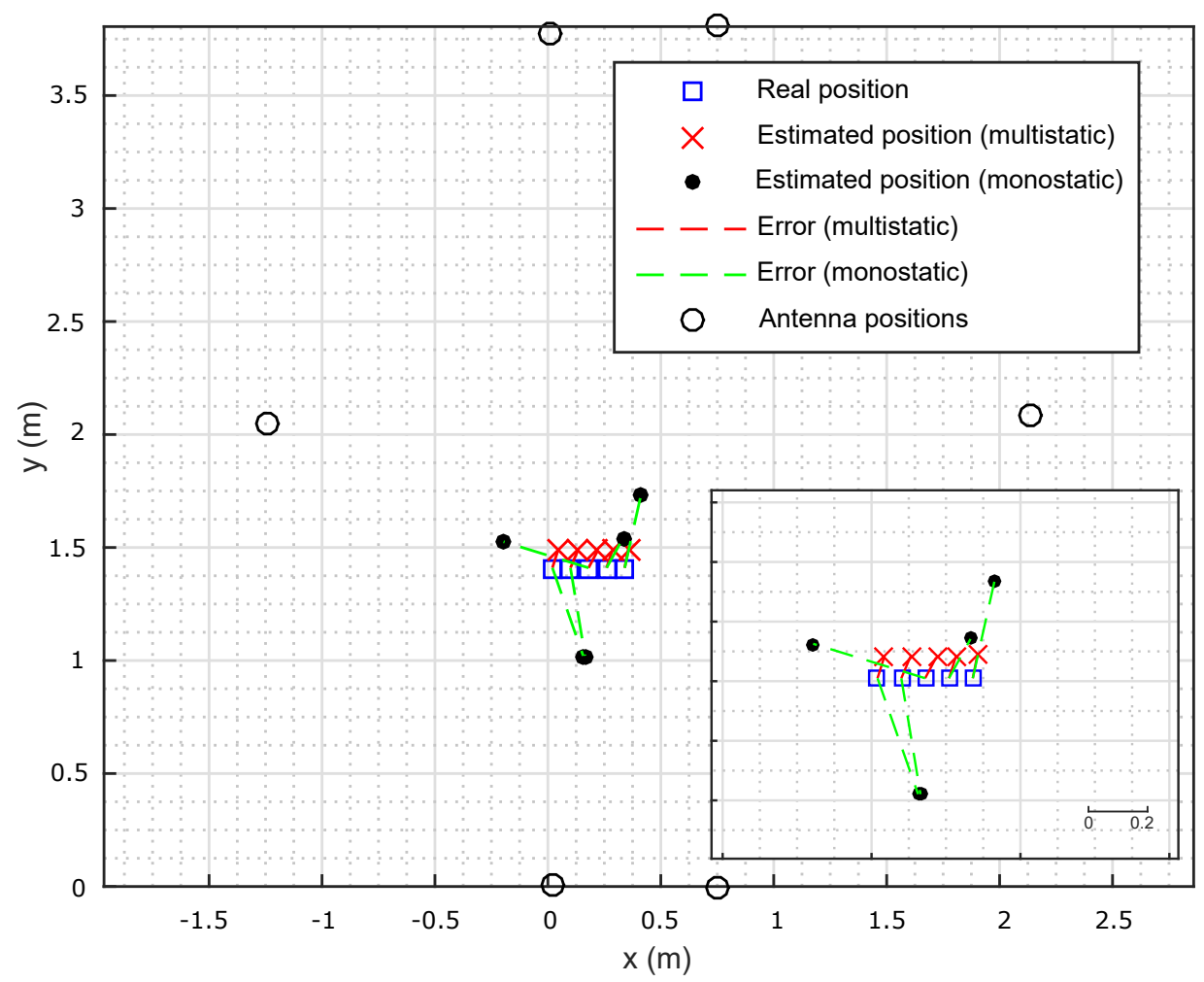

Figure 17: Real positions of the RFID tag (blue squares) and estimated positions by the multistatic (red crosses) and the monostatic (black dots) localization system. The area surrounding the real positions of the RFID tag is zommed for the sake of clearness. The antenna positions are represented with black circumferencescircles.

successfully validated for RFID tags in the UHF band, it is expected that they can be directly extended to other working bands.

In addition, the advantages of using multistatic phase measurements in some scenarios have been remarked emphasizing on the increase of the unambiguous region and the reduction of local minima due to the slower phase variation of this approach. A localization system was developed as a proof of concept to illustrate a potential use for the proposed measurement setup. The system was evaluated by means of simulation and measurements in test scenario in anechoic chamber. The results show a centimetric accuracy on the tag estimated position.

\section{Acknowledgements}

This work has been supported by the Gobierno del Principado de Asturias (PCTI)/FEDER-FSE under project GRUPIN14-114; by the Ministerio de Ciencia e Innovación of Spain/FEDER under projects TEC2014-55290-JIN and MIRIIEM-TEC2014-54005-P and under the FPU grant FPU15/06431. 
[1] GS1, EPC radio-frequency identity protocols generation-2 UHF RFID (11 2016). URL http://www.gs1.org/sites/default/files/docs/epc/Gen2_Protocol_Standard.pdf

[2] W. Alsalih, A. Alma'aitah, W. Alkhater, RFID localization using angle of arrival cluster forming, Int. J. Distrib. Sensor Networks $10(3)$.

[3] A. A. N. Shirehjini, A. Yassine, S. Shirmohammadi, An RFID-based position and orientation measurement system for mobile objects in intelligent environments, IEEE Trans. Instrum. Meas. 61 (6).

[4] Y. Alvarez, M. E. D. Cos, F. Las-Heras, A received signal strength RFID-based indoor location system, Sens. Actuators A Phys. 255 (2017) 118-133.

[5] S. Shao, R. J. Burkholder, Item-level RFID tag location sensing utilizing reader antenna spatial diversity, IEEE Sensors J. 13 (10) (2013) 3767-3774.

[6] X. Chen, L. Xie, C. Wang, S. Lu, Adaptive accurate indoor-localization using passive RFID, in: Proc. IEEE Int. Conf. Parallel Distrib. Syst. (ICPADS), 2013, pp. 249-256.

[7] P. V. Nikitin, R. Martinez, S. Ramamurthy, H. Leland, G. Spiess, K. V. S. Rao, Phase based spatial identification of UHF RFID tags, in: 2010 IEEE Int. Conf. on RFID, 2010, pp. 102-109.

[8] S. Azzouzi, M. Cremer, U. Dettmar, T. Knie, R. Kronberger, Improved aoa based localization of UHF RFID tags using spatial diversity, in: Proc. IEEE Int. Conf. RFID-Technol. Appl. (RFID-TA), 2011, pp. 174-180.

[9] J. Huiting, H. Flisijn, A. B. J. Kokkeler, G. J. M. Smit, Exploiting phase measurements of EPC Gen2 RFID tags, in: Proc. IEEE Int. Conf. RFID-Technol. Appl. (RFID-TA), 2013, pp. 1-6.

[10] R. Miesen, F. Kirsch, M. Vossiek, UHF RFID localization based on synthetic apertures, IEEE Trans. Autom. Sci. Eng. $10(3)$.

[11] A. Buffi, P. Nepa, Advanced SARFID: a localization technique for UHF RFID tags, in: URSI Int. Symp. Electromagn. Theory (EMTS), 2016.

[12] A. Buffi, P. Nepa, The SARFID technique for discriminating tagged items moving through a UHF-RFID gate, IEEE Sensors Journal 17 (9) (2017) 2863-2870.

[13] B. Wagner, N. Patwari, D. Timmermann, Passive RFID tomographic imaging for device-free user localization, in: 2012 9th Work. Positioning, Navig. Commun., 2012, pp. 120-125.

[14] M. Scherhäufl, M. Pichler, E. Schimbäck, D. J. Müller, A. Ziroff, A. Stelzer, Indoor localization of passive UHF RFID tags based on phase-of-arrival evaluation, IEEE Trans. Microw. Theory Techn. 61 (12) (2013) 4724-4729.

[15] M. Scherhäufl, M. Pichler, A. Stelzer, UHF RFID localization based on evaluation of backscattered tag signals, IEEE Trans. on Instrum. Meas. 64 (11) (2015) 2889-2899.

[16] D. D. Donno, F. Ricciato, L. Catarinucci, A. Coluccia, L. Tarricone, Challenge: Towards distributed RFID sensing with software-defined radio, in: Proc. of the Annu. 16th Int. Conf. on Mobile Comput and Netw., 2010, pp. 97-104.

[17] A. Guerra, N. Decarli, F. Guidi, D. Dardari, Energy sprinklers for passive UWB RFID, in: 2014 IEEE International Conference on Ultra-WideBand (ICUWB), 2014, pp. 356-361.

[18] Triquint Semiconductor, Saw delay line (10 2016). URL http://www.richardsonrfpd.com/resources/RellDocuments/SYS_31/856716.pdf

[19] Imping, Imping low level user data (10 2016). URL https : //support.impinj .com/hc/en-us/articles/202755318-ApplicationNote-Low-Level-User-Data-Support

[20] Y. Alvarez-Lopez, G. A. Narciandi, F. Las-Heras, Sensor network and inertial positioning hybridisation for indoor location and tracking applications, Int. J. Sensor Networks (Accepted for publication). 